\title{
Acute care in neurosurgery: quantity, quality, and challenges
}

\author{
M U Schuhmann, E Rickels, S K Rosahl, C G Schneekloth, M Samii
}

\begin{abstract}
Objective-Part of the daily routine in neurosurgery is the treatment of emergency room admissions, and acute cases from other departments or from outside hospitals. This acute care is not normally included in performance figures or budget management, nor analysed scientifically in respect of quantity and quality of care provided by neurosurgeons.

Method-Over a 1 year period, all acute care cases managed by two neurosurgical on call teams in a large northern German city, were recorded prospectively on a day by day basis. A large database of 1819 entries was created and analysed using descriptive statistics.

Results-The minimum incidence of patients requiring neurosurgical acute care was estimated to be $75-115 / 100000$ inhabitants/year. This corresponds to a mean of about 6/day. Only $30 \%$ of patients came directly via the emergency room. The fate of $70 \%$ of patients depended initially on the "neurosurgical qualification" of primary care doctors and here deficits existed. Although most intracerebral and subarachnoid haemorrhages were managed with the participation of neurosurgeons, they were not involved in the management of most mild and moderate traumatic brain injuries. Within 1 year the additional workload from acute care amounted to 1000 unplanned admissions, 900 acute imaging procedures, and almost 400 emergency operations.
\end{abstract}

Conclusion-The current policy in public health, which includes cuts in resources, transport facilities, and manpower, is not compatible with the demonstrated extent of acute neurosurgical care. In addition to routine elective work, many extra admissions, evening or night time surgery, and imaging procedures have to be accomplished. An education programme for generalists is required to improve overall patient outcome. These conclusions hold special importance if health authorities wish to not only maintain present standards but aim to improve existing deficits. (F Neurol Neurosurg Psychiatry 2001;71:182-187)

Keywords: neurosurgery; acute care; budget; education

The treatment of additional patients from the emergency room and of other hospitals or departments, who require acute care, is a part of the daily work of most neurosurgical units, which from the point of view of analysis, is widely ignored. For management purposes, hospital officials tend to rely on easily accessible performance measures such as hospital occupancy, rate of admissions to hospital, duration of stay, and number of operations each year. However, the activity of the on call team, usually staffed by surgeons working in addition to their elective duties, is not reflected in these figures. Moreover, this work is normally not recorded in detail and is therefore lost for further analysis. On the other hand important variables such as final patient outcome, the quality of care, the duration of stay in hospital, the use and misuse of resources and, consequently, reputation and economic status of a neurosurgical department, depend on every aspect of the quality of performance.

We prospectively recorded and analysed the daily work of the two neurosurgical on call teams in the City of Hannover, State Capital of Lower Saxony, Germany. The purpose of the study was to record and analyse the quantity and quality of neurosurgical on call activity in order to document deficits in the chain of treatment and to gather epidemiological data of acute neurosurgical disease of the area. The results are discussed with regard to the current public health policy of limiting or decreasing budgets, technical resources, and manpower.

\section{Methods}

The two major departments of neurosurgery in Hannover, Germany (both directed by the senior author) combine a total of 140 beds, 80 of which are in the Medical School Hannover and 60 in Nordstadt Hospital including 15 neurosurgical intensive care unit (ICU) beds. During normal working hours, the on call team consists of a resident and a senior neurosurgeon. Thereafter they are supplemented by the on call resident running the ICU (Hannover Medical School) or a resident at home who is available within 15 minutes (Nordstadt).

The city of Hannover has 522000 inhabitants with an additional 1.5 million inhabitants living in close proximity in the surrounding areas. Another 1.25 million inhabitants populate more distant regions, from which we receive about $25 \%$ of our outside patients, although other neurosurgical centres are within a comparable distance. Consequently, both neurosurgical centres cover 2-3 million people. ${ }^{1}$ A prospective 1 year study from April 1997 to April 1998 was performed. The resident on call recorded every patient that he either saw personally or that he provided neurological advice for over the telephone on a specifically designed chart consisting of 63 separate questions. Over a 1 year period charts were collected each morning and the information was entered into a computer database under the following field headings: 
- Personal data

- Date and time of first contact

- Patient origin (emergency room, different hospital, within own hospital)

- Suspected diagnosis

- Duration of symptoms

- Neurological status

- Existing images and mode of image transfer

- Neurosurgical action

- Final neurosurgical diagnosis.

Routine consultations in other hospital departments were carried out by the regular

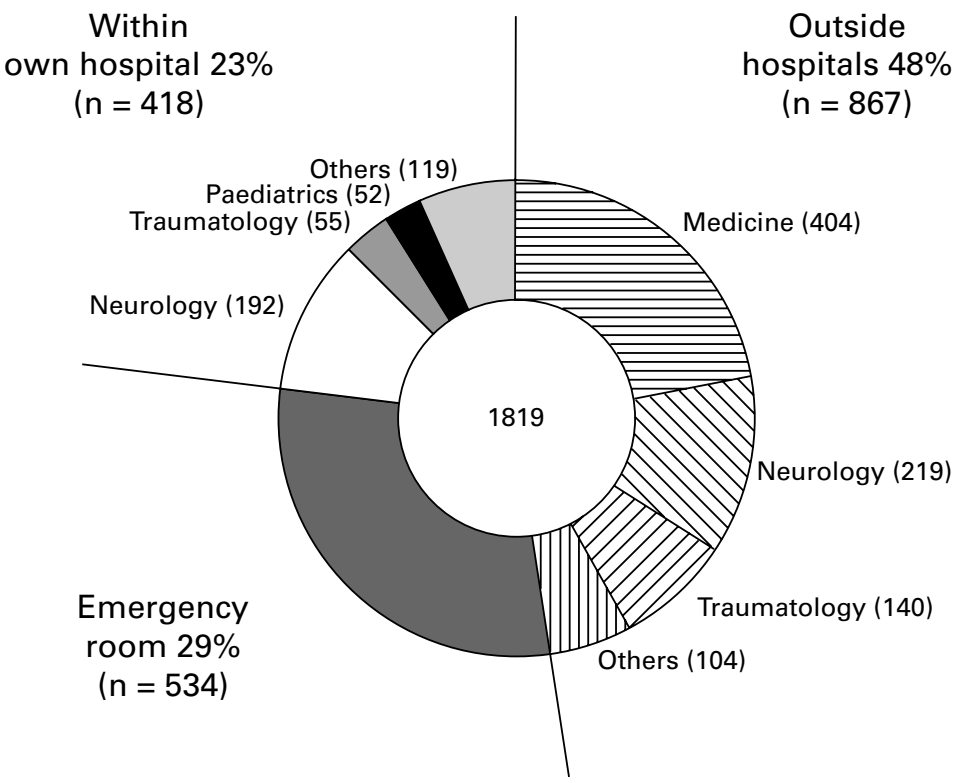

Figure 1 Distribution of the origin of 1819 recorded acute care patients. Almost $50 \%$ of patients came from other hospitals, most from internal medicine units. Only $30 \%$ of patients came directly to the emergency room, $70 \%$ of them arriving by helicopter or ambulance; $23 \%$ came from departments within our own hospitals.
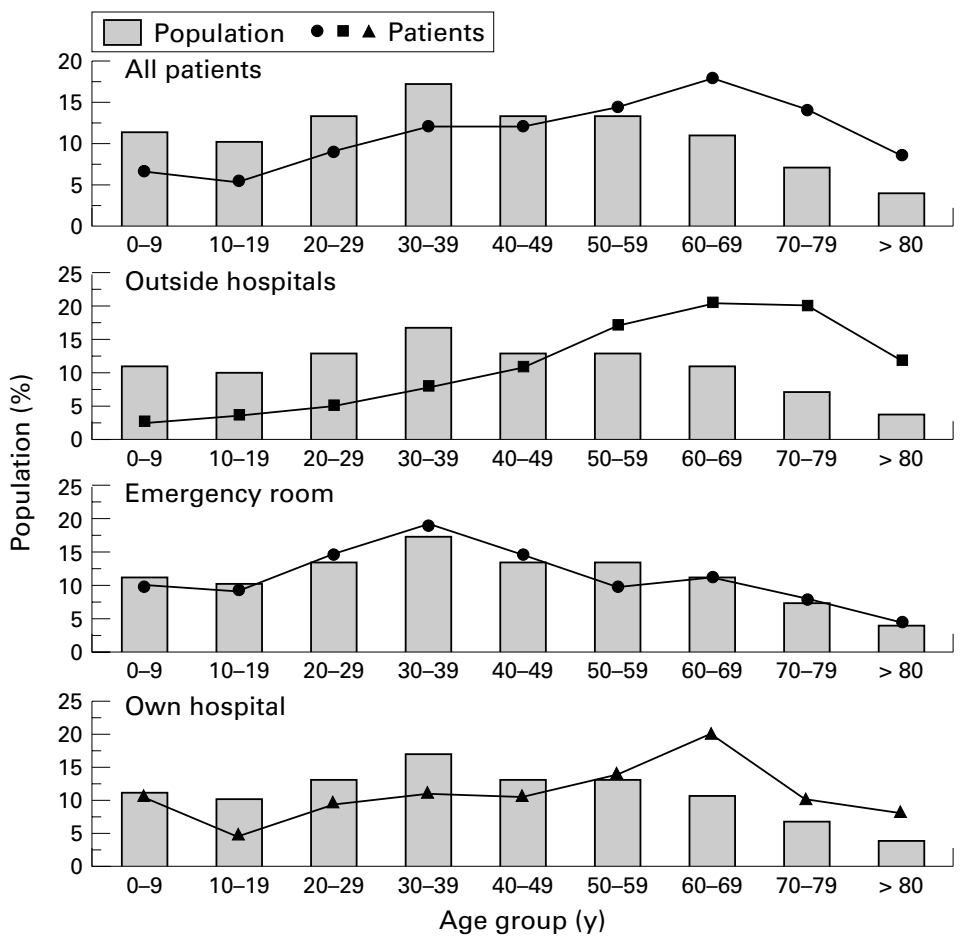

Figure 2 Distribution of age groups of all recorded patients and of patients subdivided by origin (line graphs), compared with the related population (bar graphs). consulting service and were not included in the survey. Data were analysed using descriptive tests with a standard software package (StatView ${ }^{\circledR}$, Abacus Concepts).

\section{Results}

NUMBER OF PATIENTS

The number of patient contacts recorded over a 1 year period was 1819,1441 at Hannover Medical School and 376 at Nordstadt. The actual number of patient contacts was higher because not all days were sufficiently monitored. Based on the number of days with complete records, we estimate a total of 2300 patients/year with an average of 4.2 patients/ day at Hannover Medical School and 2.2 patients/day at Nordstadt. From 2-3 million inhabitants there was an average of at least six patients/day in immediate need of a neurosurgeon. The incidence of neurosurgical acute care cases was accordingly 76-114/100 000 inhabitants/year.

\section{CONTACTS}

Figure 1 shows the distribution of patient contacts.

\section{AGE}

Figure 2 displays the age distribution of the recorded patients in relation to the distribution of the population. The incidence of acute neurosurgical illness in the population was proportionally less in the age groups younger than 40 ( $32 \%$ of all cases in $51 \%$ of the population) and more in the age groups older than 50 (56\% of all cases in $35 \%$ of the population). This result relates to the age distribution of patients from smaller outside hospitals, the largest source of acute care patients. Patients seen in our emergency room, however, closely reflected the age distribution of the general population.

\section{REFERRING AND FINAL NEUROSURGICAL}

DIAGNOSIS

The patients were grouped according to the final neurosurgical diagnosis as follows:

- Traumatic brain injury

- Intracerebral haemorrhage

- Neoplastic diseases

- Spinal pathology

- Subarachnoid haemorrhage

- Hydrocephalus associated illness

- Chronic subdural haematoma

- Infection

- Miscellaneous.

Table 1 displays the patient distribution. A quarter of the patients had traumatic brain injury, another quarter had intracranial haemorrhage and of these, every fourth was a subarachnoid haemorrhage. Less than 10\% were seen because of spinal pathology (inflammatory disease excluded). Subarachnoid haemorrhage was the most precisely recognised disease, all other diseases, except for infection and hydrocephalus associated illness, were correctly diagnosed in more than $90 \%$ of cases. However, referring doctors misdiagnosed hydrocephalus associated illness in almost a third of patients. 
Table 1 Incidence of diseases according to final diagnosis and accuracy of referring diagnosis

\begin{tabular}{lccclc}
\hline $\begin{array}{l}\text { Diagnosis } \\
\text { group }\end{array}$ & $\begin{array}{c}\text { Recorded } \\
\text { patients }(n)\end{array}$ & $\begin{array}{l}\text { Etimated } \\
\text { total }(n)^{\star}\end{array}$ & Total (\%) & $\begin{array}{l}\text { Incidencet } \\
\text { (number/100000 } \\
\text { inhabitants/y) }\end{array}$ & $\begin{array}{l}\text { Deviant } \\
\text { referring } \\
\text { diagnosis (\%) }\end{array}$ \\
\hline TBI & 484 & 583 & 26.6 & $19-29$ & 5.6 \\
ICH & 320 & 412 & 17.6 & $14-21$ & 4.4 \\
ND & 222 & 303 & 12.2 & $10-15$ & 7.7 \\
SP & 168 & 235 & 9.2 & $8-12$ & 7.7 \\
SAH & 119 & 147 & 6.5 & $5-7$ & 2.5 \\
HAI & 107 & 121 & 5.9 & $4-6$ & 6.2 \\
cSDH & 81 & 101 & 4.5 & $3-5$ & 12.5 \\
INF & 40 & 52 & 2.2 & $2-3$ & \\
MSC & 278 & 333 & 15.3 & $11-17$ & \\
Total & 1819 & 2287 & 100 & $76-114$ & \\
\end{tabular}

TBI=Traumatic brain injury; ICH=intracerebral haemorrhage; ND=neoplastic diseases; $\mathrm{SP}=$ spinal pathology; $\mathrm{SAH}=$ subarachnoid haemorrhage; $\mathrm{HAI}=$ hydrocephalus. $\mathrm{cSDH}=$ chronic subdural haematoma; INF=infection; $\mathrm{MSC}=$ miscellaneous.

^Estimated according to fully recorded days.

tCalculated on the basis of estimated total number of cases/year.

Figure 3 displays the relative age distribution within each patient group in comparison with the population.

\section{PATIENT DISTRIBUTION OVER YEAR, WEEK, AND} DAY

Relatively more patients were seen from May to September (160-190/month compared with 106-130/month October to April). June and July were peak months in the emergency room, reflecting that most cases of traumatic brain injury were recorded in this time (77 and $80 /$ month). intracerebral haemorrhage showed peaks in spring and autumn, whereas subarachnoid haemorrhage and neoplastic diseases were evenly distributed over the year. In general, more patients were seen on Fridays and Saturdays. More patients originating from outside hospitals were seen on Fridays whereas those from within our hospitals were most frequent on Saturdays. The number of patients admitted to the emergency room was evenly distributed
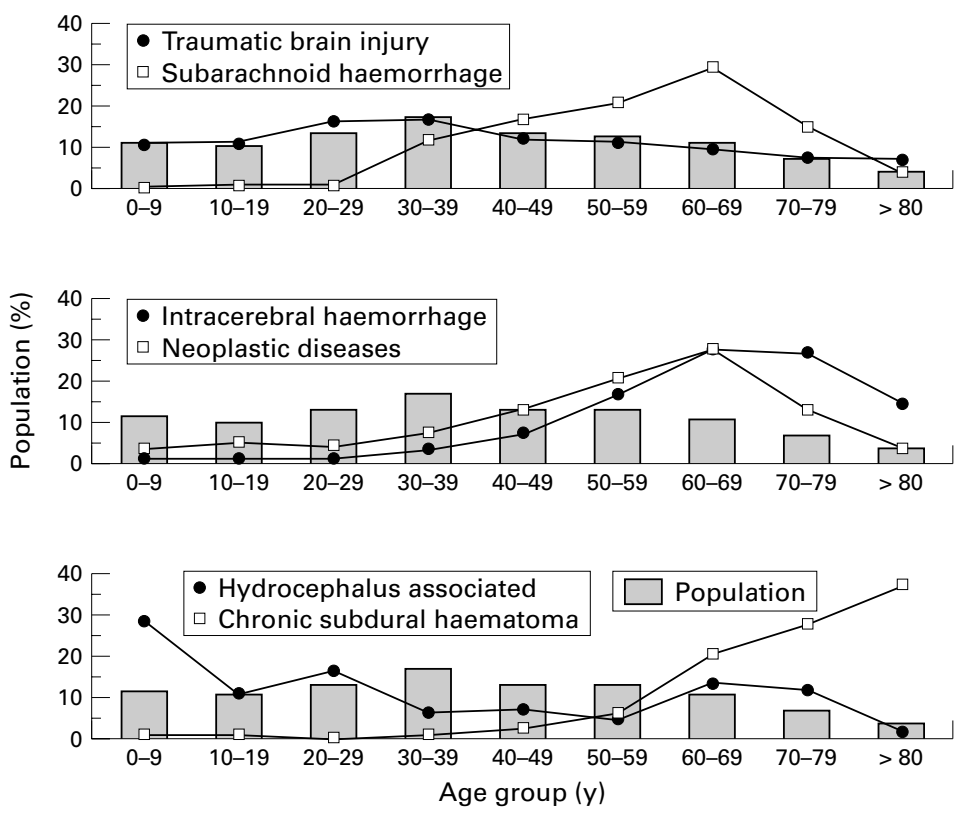

Figure 3 Distribution of age groups of the related population in comparison with patients subdivided by disease. Traumatic brain injury had an equal impact on all age groups. Neoplastic disease and intracranial haemorrhage (intracerebral and subarachnoid haemorrhage) are predominantly diseases of those older than 50 years. Hydrocephalus associated disease peaked in children below 10 years, chronic subdural haematomas in patients above 80 years.

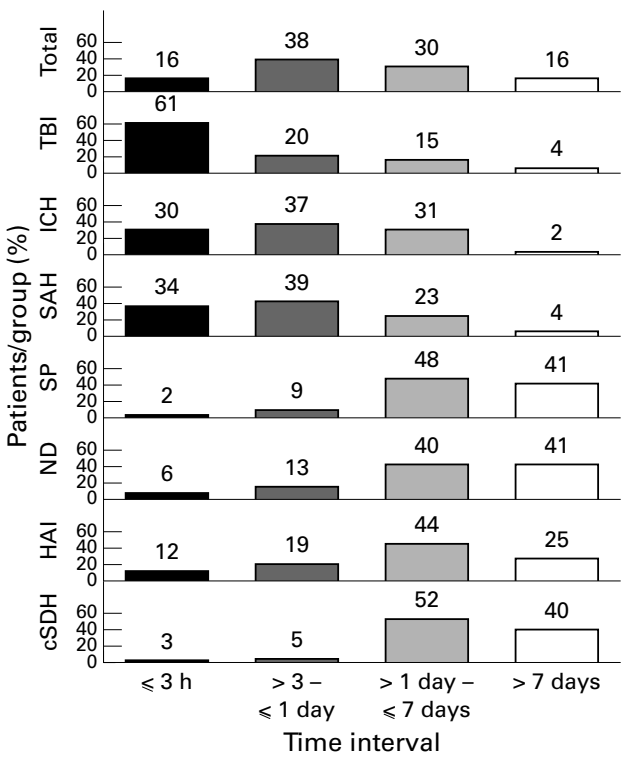

Figure 4 Time intervals between onset of symptoms and first contact with the neurosurgeon. Only about $15 \%$ of all contacts with a neurosurgeon took place in the first 3 hours, little more than 50\% were established 24 hours after onset of symptoms. In $67 \%$ to $81 \%$ cases of intracerebral haemorrhage (ICH), subarachnoid haemorrhage ( $S A H)$, and traumatic brain injury (TBI) contacts were made within 1 day. For TBI $61 \%$ of all contacts were established within 3 hours. By contrast, in $40 \%-50 \%$ of cases of neoplastic disease (ND), spinal pathology (SP), and chronic subdural haematoma (cSDH) 2-7 days elapsed after the onset of symptoms before a neurosurgeon was contacted. In a further $40 \%$ the delay was for more than a week. HAI=hydrocephalus associated illness.

over the week. Patients with traumatic brain injury peaked on Sundays. Less than $50 \%$ of recorded patients were seen by $500 \mathrm{pm}$ with most patients being seen up until $200 \mathrm{am}$. Most patients within our own hospitals were recorded between $600 \mathrm{pm}$ and $900 \mathrm{pm}$.

TIMING OF CALL FOR NEUROSURGEON

Figure 4 displays the elapsed time from the beginning of the symptoms until a neurosurgeon was approached.

NEUROLOGICAL CONDITION

A simple neurological status was recorded and patients were graded according to the Glasgow coma scale (GCS) on admission or at the time of contact if they remained in outside hospitals (table 2).

IMAGING

Neuroimaging existed in 1202 of 1819 patients $(66 \%)$ by the time of first contact with the neurosurgeon. Seven hundred and twenty three of these 1202 patients were in outside hospitals. In $69 \%$ of cases an explanation of the radiological findings over the telephone was sufficient for a decision about further action. In 104 cases $(14 \%)$ images were sent by taxi and image transfer via video mail box (VP2000) or video on line conference (MEDCOM) was used to guide further management in 123 cases $(17 \%)$.

Of 1361 patients seen by the on call teams, $64 \%$ had already been imaged. Three hundred and twenty four patients received a further 353 imaging procedures ( $57 \%$ CT scans) to 
Table 2 Neurological condition of patients at time of admission*

\begin{tabular}{lcccc}
\hline Criteria & Total (\%) & TBI (\%) & ICH (\%) & SAH (\%) \\
\hline GCS 3 & 23 & 42 & 27 & 29 \\
GCS 4-8 & 8 & 7 & 17 & 11 \\
GCS 9-13 & 15 & 12 & 26 & 16 \\
GCS 14-15 & 54 & 39 & 30 & 43 \\
Ventilated at GCS 3 & & 100 & 97 \\
Ventilated at GCS 4-8 & 64 & 35 & 62 \\
Unilaterally fixed/dilated pupil & & 8 & 8 & 8 \\
Bilaterally fixed/dilated pupil & & 5 & 12 & 4 \\
?1 corneal reflex absent & 10 & 48 & 13 \\
Hemiparesis & & & 25 \\
Hunt and Hess I & & & 18 \\
Hunt and Hess II & & & 18 \\
Hunt and Hess III & & & 21 \\
Hunt and Hess IV & & & \\
Hunt and Hess V & & &
\end{tabular}

${ }^{\star}$ Or contact if not transferred; GCS=Glasgow coma score ${ }^{15}$; TBI=patients with traumatic brain injury; $\mathrm{ICH}=$ patients with intracerebral haemorrhage; $\mathrm{SAH}=$ patients with subarachnoid haemorrhage.

complete the investigation. The 491 patients arriving without any imaging required a total of 528 imaging procedures to be performed. In total, $598 \mathrm{CT}$ scans, $50 \mathrm{MR}$ scans, 77 digital substraction angiographies, and 156 plain radiographs (excluding preoperative routine chest radiographs) were initiated by the on call team.

NEUROSURGICAL ACTION

Neurosurgical action is outlined in figure 5. Seventy five per cent of all patients were personally seen by a neurosurgeon. Of 1361 patients examined by a neurosurgeon $64 \%$ were admitted or transferred from another service to a neurosurgical ward. Every second admission carried an indication for surgical intervention. Twenty five per cent of patients remained on their non-neurosurgical wards, only $10 \%$ had an elective indication for later surgery. The remaining $11 \%$ of the patients

Neurosurgical action
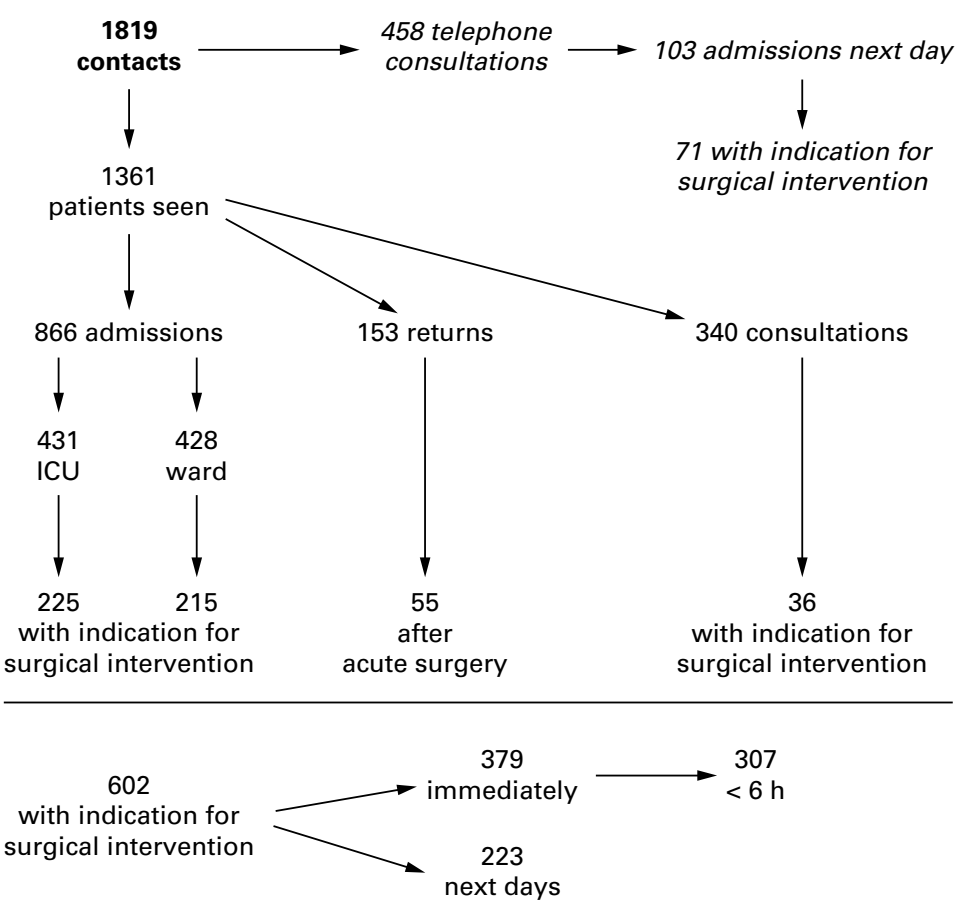

Figure 5 Flow sheet of neurosurgical action after establishment of first contact. were sent back to an outside hospital, a third of them immediately after surgical treatment.

One third of the overall 1819 patient contacts required surgical treatment and in $62 \%$ this was performed immediately by the on call team, in most cases within 6 hours. Forty six per cent of acutely operated patients were admitted to an ICU and a further $15 \%$ were returned to their outside hospitals due to the ICU reaching full capacity. A bed on the neurosurgical ICU was available for only $50 \%$ of the total ICU admissions.

Figure 6 displays the different neurosurgical actions and indications for surgery for patients with traumatic brain injury, intracerebral haemorrhage, or subarachnoid haemorrhage.

\section{Discussion}

The survey succeeded in creating a databank with more than 1800 patient contacts and 114 500 data entries, representing $80 \%$ of all neurosurgical acute care and emergency cases that had been managed at the two major neurosurgical units in Hannover within 1 year. The minimum number of inhabitants that required the opinion or the personal treatment of a neurosurgeon within minutes to a few hours was 70-115 people/100 000 inhabitants/ year

Although the most obvious neurosurgical emergencies - traumatic brain injury and intracranial haemorrhage (intracerebral haemorrhage and subarachnoid haemorrhage), were the most common disease groups in our material-they represented just $50 \%$ of all cases.

The incidence of traumatic brain injury (19$29 / 100000$ people/year) in our material, is certainly very low compared with an overall incidence of 200/100 000 people/year for the United States. ${ }^{2}$ The major reason is that minor head injuries, accounting for about $80 \%$ of all head injuries, are never seen by a neurosurgeon after being admitted to a peripheral hospital. About $10 \%$ of patients with traumatic brain injury die before they reach hospital. ${ }^{3}$ We did not find any specific age group predominantly at risk of sustaining traumatic brain injury. Investigations from the United States, however, describe traumatic brain injury as " .. . predominantly a disease of young adult men ..." and found a peak incidence in the age range of 20 to 30 years. $^{3-5}$ On the other hand, the age distribution of our patients correlates well with the results of the European brain injury consortium survey of moderate to severe traumatic brain injury. ${ }^{6}$

For subarachnoid haemorrhage the presumed incidence in the population of 5-7/ 100000 people/year correlates with figures from comparable western countries ranging from 5-8/100 000 people/year. ${ }^{7-11}$

In the present survey intracerebral haemorrhage was two to three times more common than subarachnoid haemorrhage. The high percentage of telephone consultations for intracerebral haemorrhage resulting in conservative treatment $(66 \%)$ creates the impression that colleagues from outside hospitals obtained a neurosurgical opinion for most 

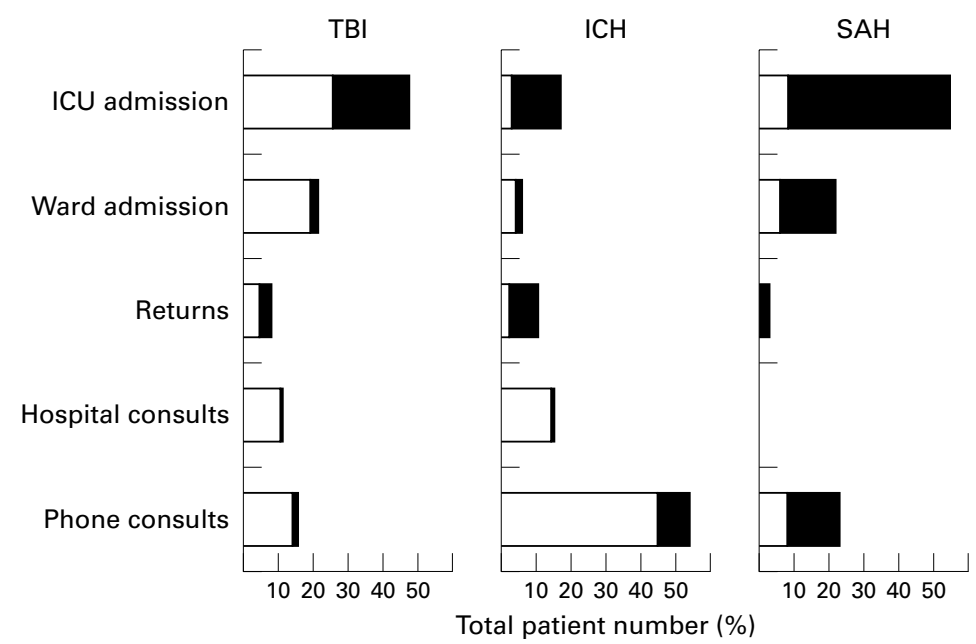

Conservative

Surgery

Figure 6 Differences in neurosurgical action and indications for surgery for traumatic brain injury (TBI), intracerebral haemorrhage (ICH), and subarachnoid haemorrhage $(S A H)$. About $50 \%$ of patients with TBI or SAH were immediately admitted to an ICU, whereas about $50 \%$ of all patients with $\mathrm{ICH}$ were managed by telephone consultation. Most (78\%) patients with ICH had no indication for surgery. In about $74 \%$ of cases of TBI conservative treatment was indicated. However, $78 \%$ of patients with SAH carried an indication for surgery; $25 \%$ of SAH contacts were not admitted. In a third of these cases no ICU bed was available, one third of patients were graded Hunt and Hess $V$ with fixed and dilated pupils, and for one third admission was planned the next morning

cases of intracerebral haemorrhage. As the overall incidence correlates well with the literature, ${ }^{12}$ we assume that most patients with intracerebral haemorrhage and subarachnoid haemorrhage were managed with the participation of neurosurgeons.

The peripheral hospitals provided $50 \%$ of all patients, most of whom were elderly and thus dominated the overall age distribution within the study. By contrast, the emergency room admissions reflected the age distribution of the general population. One possible reason for this difference is that the available on call neurosurgeon becomes involved in every domestic "neurocase", whereas patients from outside hospitals are often only transferred in severe cases. With the exception of traumatic brain injury, these are predominantly diseases of elderly people; $73 \%$ of intracerebral haemorrhage, $74 \%$ of subarachnoid haemorrhage, and $75 \%$ of chronic subdural haematoma came from outside hospitals.

As only $30 \%$ of all patients came directly to our emergency rooms, primary care colleagues with no training in neurosurgery or neurology handled the first contact with the medical system for the vast majority of cases. Therefore most patients' fate was not only dependent on the capacity and quality of the neurosurgical centre, but equally on the quality and knowledge of primary care doctors. The ability to correctly identify the cause of the patient's distress was aided by obvious symptoms and a clear cut history. The rate of less than 10\% incorrect diagnoses for traumatic brain injury, subarachnoid haemorrhage, intracerebral haemorrhage, neoplastic diseases, spinal pathology, and chronic subdural haematoma is encouraging and based on the availability of CT in almost all peripheral hospitals. A known hydrocephalus, however, created insecurity in differentiating true shunt dysfunction from other diseases (29\% incorrect diagnoses).

Key features, besides a correct diagnosis, are comprehension and timing of neurosurgical treatment concepts. Here we encountered further deficits; $27 \%$ of contacts after subarachnoid haemorrhage, $33 \%$ after intracerebral haemorrhage, and 19\% after traumatic brain injury were established later than 24 hours after onset of symptoms, which in most cases were conclusive. Diseases with a more insidious onset and slower progression of symptoms had even longer delays (fig 4). The peak in the number of recorded patients from outside hospitals on Fridays points to the same problem: as the weekend approaches doctors try to transfer patients with unsolved problems, although this could have been done earlier. Similarly, most patients in other departments within our own institution were seen on Saturdays.

A teaching programme, run by the national neurosurgical society or regionally by the centre itself, is therefore necessary and overdue to counterbalance the existing deficits in the periphery. We would expect a substantial increase in the overall quality of neurosurgical acute care and a decrease in costs, if unnecessary transports and delays, and therefore prolonged treatment, could be avoided.

For the management of cases requiring neurosurgical acute care, the following facts need to be highlighted. Over a 1 year period, there was a demand for at least 600 operations in addition to the elective cases, for at least 500 unplanned admissions to ICUs and 500 to normal wards, and for at least 900 imaging procedures. Fifty per cent of the on call team's work had not even started before $500 \mathrm{pm}$. In addition, all members of the on call team had to accomplish their personal part of elective operative cases and routine ward work. Consequently there is the need for a dedicated team, incorporating more than just two on call neurosurgeons. These people have to be highly qualified to accomplish for example, telephone consultations, which first year juniors are not able to handle efficiently. The team needs to be well staffed to cover for the additional working load. A neuroanaesthesiology team needs to be assigned and the working hours of theatre staff at night have to be compensated by others during the next day. A neuroradiology team providing 24 hour CT, angiography, and MRI also have to be available. Concerning the ICU capacity, there is, as in other European countries, a desperate need for neurosurgical beds. ${ }^{13}{ }^{14}$ In the present study, only $50 \%$ of all ICU admissions could be directed to the neurosurgical units. Fifty five postoperative patients had to return to outside hospitals because no ICU bed was available. Thirty patients with traumatic brain injury, subarachnoid haemorrhage, or intracerebral haemorrhage could not be treated at all because there were no theatre or ICU capacity available. A considerable number of those patients that either returned to outside hospitals or could 
not be accepted, had been refused by other neurosurgical centres already.

Acute care work deserves more attention than it is receiving at present with regard to the current demands for quality control on the one hand and increased cutting of financial resources, numbers of medical personnel, and beds on the other hand. Additionally, European laws will impose stricter regulations on the maximum number of working hours allowed. The requirements for acute care in neurosurgery, as demonstrated by the present study, as well as the obligation to maintain a high standard of medical care for the population and to improve existing deficits, are not compatible with this development. Increases in budget, facilities, and staff numbers are required and have to be demanded on the political floor on the basis of studies such as the present one.

We thank all of our colleagues who have made this study possible by recording their daily on call work in addition to their many other duties. We are grateful to Mrs Eleni Petridou for entering the data into the database, and to Dr Neil G Harris for his revision of the English in this manuscript.

1 Niedersächsiches Landesamt für Statistik. Bevölkerung nach Altersgruppen. Hannover: Lanc Niecersachen 1996.

2 Francel P, Alves WM, Jane JA. Mild head injury in adults. In: Youmans JR, ed. Neurological surgery. Philadelphia: Wilkins and Wilkins, 1996:

3 Kelly DF, McBride DQ, Becker DP. Surgical management of severe closed head injury in adults. In: Schmidek $\mathrm{HH}$,
Sweet WH, eds. Operative neurosurgical techniques. Philadelphia: WB Saunders, 1995:47-67.

4 Kraus JF, McArthur DL. Epidemiology of brain injury. In: Cooper PR, Golfinos JG, eds. Head Injury. Baltimore: Williams and Wilkins 2000:1-26.

5 Marshall LF, Gautille T, Klauber MR. The outcome of severe closed head injury. $\mathcal{F}$ Neurosurg 1991;75:28-36.

6 Murray GD, Teasdale GM, Braakman R, et al. The European Brain Iniury Consortium survey of head injuries. Acta Neurochir (Wien) 1999;141:223-36.

7 Linn FH, Rinkel GJ, Algra A, et al. Incidence of subarachnoid hemorrhage: role of region, year, and rate of computed tomography: a meta-analysis. Stroke 1996;27: 625-9.

8 Chyatte D, Chen TL, Bronstein K, et al. Seasonal fluctuations in the incidence of intracranial aneurysm rupture and its relationship to changing climatic conditions. $\mathcal{F}$ Neurosurg 1994;81:525-30.

9 Bamford J, Sandercock P, Dennis M, et al. A prospective study of acute cerebrovascular disease in the community: the Oxfordshire Community Stroke Project 1981:86. 2. Incidence, case fatality rates and overall outcome at 1 year of cerebral infarction, primary intracerebral and subarachnoid haemorrhage. F Neurol Neurosurg Psychiatry 1990;53: $16-22$.

10 Harmsen P, Tsipogianni A, Wilhelmsen L. Stroke incidence rates were unchanged, while fatality rates declined, during 1971-87 in Goteborg, Sweden. Stroke 1992;23:1410-15.

11 Herman B, Leyten AC, van Luijk JH, et al. Epidemiology of stroke in Tilburg, the Netherlands. The population- based stroke incidence register: 2. Incidence, initial clinical picture and medical care, and 3-week case fatality. Stroke 1982;13:629-34.

12 Broderick JP, Brott T, Tomsick T, et al. Intracerebral hemorrhage more than twice as common as subarachnoid hemorrhage. $\mathcal{F}$ Neurosurg 1993;78:188-91.

13 Crimmins DW, Palmer JD. Snapshot view of emergency neurosurgical head injury care in Great Britain and Ireland. F Neurol Neurosurg Psychiatry 2000;68:8-13.

14 Cupples PA, Makin AP, McKinnon SS, et al. More intensive care unit beds are needed. BMF 1997;314:351.

15 Teasdale G, Jennett B. Assessment of coma and impaired consciousness: a practical scale. Lancet 1974;ii:81. 